\title{
PERFIL SOCIODEMOGRÁFICO E CLÍNICO DE IDOSOS SUBMETIDOS À HEMODIÁLISE
}

\author{
Ana Elza Oliveira de Mendonça', Jessicleide da Guia Dantas², Débora Azevedo de Andrade², \\ Camila Tedeschi Segato ${ }^{2}$, Gilson de Vasconcelos Torres ${ }^{3}$
}

${ }^{1}$ Enfermeira. Doutoranda Ciências da Saúde. Universidade Federal do Rio Grande do Norte. Natal-RN- Brasil. ${ }^{2}$ Discente em Enfermagem. Centro Universitário FACEX. Natal-RN-Brasil.

${ }^{3}$ Enfermeiro. Pós-Doutor em Enfermagem. Universidade Federal do Rio Grande do Norte. Natal-RN-Brasil.

RESUMO: Pesquisa descritiva, transversal que visou caracterizar os pacientes idosos submetidos à terapia hemodialítica, quanto aos aspectos sociodemográficos e clínicos, em dois centros de nefrologia da cidade de Natal, nordeste do Brasil, no período de janeiro a março de 2014. Participaram do estudo 81 pacientes com idade igual ou superior a 60 anos. O projeto obteve aprovação de Comitê de Ética. Por meio de formulário estruturado identificou-se que a média de idade dos pacientes foi de 70,2 anos $( \pm 7,4), 60,5 \%$ eram casados e $81,5 \%$ com ensino fundamental completo ou incompleto. A média de tempo em diálise foi de 3,5 anos $( \pm 2,2)$, 48,1\% eram hipertensos, $11,1 \%$ diabéticos e 34,6\% apresentavam associação das duas comorbidades clínicas. O desenvolvimento de doença renal crônica e a dependência de tratamento hemodialítico em pacientes idosos representam desafios aos gestores de saúde, profissionais e familiares.

DESCRITORES: Insuficiência renal crônica; Diálise renal; Enfermagem.

\section{SOCIO-DEMOGRAPHIC AND CLINICAL PROFILE OF OLDER ADULTS RECEIVING HEMODIALYSIS}

\begin{abstract}
This descriptive, transversal research aimed to characterize the elderly patients receiving hemodialysis therapy, in relation to socio-demographic and clinical aspects, in two nephrology centers in the city of Natal in the Northeast of Brazil, in January - March 2014. A total of 81 patients aged 60 years old or over participated in the study. The study was approved by the Ethics Committee. Through a structured questionnaire, it was identified that the patients' mean age was 70.2 years old $( \pm 7.4)$, $60.5 \%$ were married and $81.5 \%$ had either completed or not junior high school. The mean time in dialysis was 3.5 years $( \pm 2.2), 48.1 \%$ were hypertensives, $11.1 \%$ were diabetics, and $34.6 \%$ presented association of the two clinical comorbidities. The development of chronic kidney disease and dependence on hemodialysis treatment in elderly patients represent challenges to health managers, professionals and family members.
\end{abstract}

DESCRIPTORS: Chronic renal failure; Renal dialysis; Nursing.

\section{PERFIL SOCIODEMOGRÁFICO Y CLÍNICO DE ANCIANOS SOMETIDOS A HEMODIÁLISIS}

RESUMEN: Investigación descriptiva, transversal que tuvo como objetivo caracterizar los pacientes ancianos sometidos a la terapia hemodialítica, cuanto a los aspectos sociodemográficos y clínicos, en dos centros de nefrología de la ciudad de Natal, nordeste de Brasil, en periodo de enero a marzo de 2014. Participaron del estudio 81 pacientes con edad igual o superior a 60 años. El proyecto fue aprobado por el Comité de Ética. Por medio de formulario estructurado, se identificó que la media de edad de los pacientes fue de 70,2 años $( \pm 7,4), 60,5 \%$ eran casados y $81,5 \%$ tenían enseñanza básica completa o incompleta. La media de tiempo en diálisis fue de 3,5 años $( \pm 2,2), 48,1 \%$ eran hipertensos, $11,1 \%$ diabéticos y $34,6 \%$ presentaban asociación de los dos comorbilidades clínicas. El desarrollo de enfermedad renal crónica y la dependencia de tratamiento hemodialítico en pacientes ancianos representan desafíos a los gestores de salud, profesionales y familiares.

DESCRIPTORES: Insuficiencia renal crónica; Diálisis renal; Enfermería.
Autor Correspondente:

Ana Elza Oliveira de Mendonça

Universidade Federal do Rio Grande do Norte

Rua Coronel Milton Freire, 2821 - 50078-310 - Natal-RN-Brasil

E-mail: a.elza@uol.com.br
Recebido: 09/07/2014

Finalizado: 26/11/2014 


\section{INTRODUÇÃO}

O envelhecimento atinge cada vez mais os países em desenvolvimento e atualmente, cerca de $60 \%$ das pessoas idosas, definidas como aquelas com idade igual ou acima de 60 anos, residem nesses países. A estimativa brasileira para 2025 é que $75 \%$ da população estejam compreendidas nessa faixa etária ${ }^{(1)}$.

No Brasil foi instituído em $1^{\circ}$ de outubro de 2003 e entrou em vigor em janeiro de 2004 o Estatuto do Idoso. De acordo com a Lei ${ }^{\circ}$ 10.741, Art. $1^{\circ}$ destina-se a regular os direitos assegurados às pessoas com idade igual ou superior a sessenta anos no país ${ }^{(2)}$.

O envelhecer é único para cada ser humano, podendo o idoso possuir diferentes posturas de viver diante deste processo. Sabe-se que envelhecer faz parte da vida e é nessa etapa que, em decorrência da alta idade cronológica, ocorrem modificações de ordem biopsicossociais que afetam a relação do indivíduo com o meio ${ }^{(3)}$.

O envelhecimento populacional representa, por um lado, o êxito da saúde pública, capaz de modificar a mortalidade e aumentar a expectativa de vida, porém, economicamente, representa significativo impacto na política financeira, pois o envelhecimento está acompanhado por maior prevalência de doenças crônicas não transmissíveis, dentre as quais se destacam as doenças cardiovasculares, hipertensão arterial, Diabetes Mellitus, doença renal crônica e neoplasias que necessitam de muitos e complexos procedimentos diagnósticos, tratamentos prolongados e, consequentemente, maiores ônus aos cofres públicos ${ }^{(1)}$.

Particularmente, a Doença Renal Crônica (DRC) com número cada vez maior de pacientes que dependem de terapias de substituição da função renal para sobreviver gera grande impacto econômico e social, tornando evidente a necessidade de implementação de estratégias de promoção e prevenção na rede básica de atenção à saúde. A DRC constitui causa importante de morbidade e mortalidade e, sem dúvida, questão peculiar de Saúde Pública ${ }^{(4)}$.

De acordo com dados do censo da Sociedade Brasileira de Nefrologia (SBN), realizado em 2013, estima-se que 100.397 pessoas sejam portadoras de doença renal em tratamento dialítico. Em relação à prevalência de pacientes com mais de 60 anos verificou-se aumento de 70.872 casos em 2006 para 92.091 em 2010. Este aumento se deve em parte à melhoria da qualidade dos procedimentos dialíticos, com consequente redução da mortalidade. Cabe ressaltar que no Brasil o Sistema Único de Saúde (SUS) custeia o tratamento de $90,8 \%$ dos pacientes que necessitam de terapias renais substitutivas ${ }^{(5)}$.

Estudo destaca que as alterações morfofuncionais relacionadas ao envelhecimento humano dos rins são amplas e ocorrem de forma semelhante em outros órgãos e sistemas do corpo humano. No entanto, o caráter silencioso da doença renal, que se instala de forma lenta e progressivamente, torna o entendimento destas alterações de estrutura e função cruciais para o diagnóstico precoce em pacientes idosos. Contudo, há escassez de estudos relacionados ao grau de função renal em idosos ${ }^{(6)}$.

A relevância desta pesquisa reside em auxiliar na identificação de falhas ou lacunas, que possam estar contribuindo para o diagnóstico tardio da doença renal no idoso. Outro aspecto relevante é que poderá contribuir para melhor conhecimento da situação dos idosos portadores de doenças renais crônicas em tratamento dialítico, entre usuários de serviços públicos.

Frente ao exposto, objetivou-se caracterizar pacientes idosos submetidos à terapia hemodialítica quanto aos aspectos sociodemográficos e clínicos.

\section{MÉTODO}

Estudo descritivo e transversal, realizado de janeiro a março de 2014 em dois centros de nefrologia, situados na região metropolitana da cidade do Natal, nordeste do Brasil. Os serviços de diálise pesquisados são do Sistema Único de Saúde (SUS) e disponibilizam atendimento nas modalidades de hemodiálise e diálise peritoneal a pacientes de todas as idades. Para isso, dispõem de equipes multiprofissionais compostas por médicos e enfermeiros especialistas na área de nefrologia, assistente social, psicólogo e nutricionista.

Para a seleção da amostra foram adotados os seguintes critérios de inclusão: estar cadastrado no sistema computadorizado da unidade de diálise para o tratamento de hemodiálise, ambos os sexos, idade igual ou superior a 60 anos. 
Foram excluídos os pacientes que mesmo estando cadastrados estavam afastados do tratamento no período de coleta de dados, bem aqueles que apresentavam dificuldade de comunicação.

Foi considerada idosa a pessoa com idade igual ou superior a 60 (sessenta) anos, de acordo com a Lei $\mathrm{n}^{\circ} 10.741$, de $1^{\circ}$ de Outubro de 2003, do Estatuto do Idoso ${ }^{(2)}$. Nesse sentido, 85 idosos atenderam aos critérios de inclusão do estudo, porém quatro estavam hospitalizados durante o período de coleta de dados. Logo, a amostra foi composta por 81 idosos.

$\mathrm{O}$ instrumento de coleta de dados constou de um formulário estruturado que continha na primeira parte questões sobre características sociodemográficas e na segunda os aspectos clínicos. Cabe ressaltar que o formulário foi previamente submetido a um pré-teste com 10 idosos em outro centro de nefrologia da região metropolitana, para avaliar a aplicabilidade.

Os participantes do estudo foram entrevistados individualmente após a leitura e assinatura do Termo de Consentimento Livre e Esclarecido, atendendo aos preceitos éticos preconizados para pesquisas envolvendo seres humanos ${ }^{(7)}$. O projeto foi apreciado e aprovado pelo Comitê de Ética do Hospital Universitário Onofre Lopes (HUOL) sob o registro 233.953 e CAAE: 01094212.8.0000.5292.

Os dados obtidos foram digitalizados em planilhas do programa Microsoft Excel XP e submetidos a tratamento estatístico descritivo. Os dados foram expressos por médias, valores mínimos e máximos e desvios-padrão. O resultado da pesquisa está apresentado na sequência, por meio de tabelas.

\section{RESULTADOS}

No universo de 166 idosos cadastrados, para o tratamento de hemodiálise nos centros pesquisados, a amostra da pesquisa correspondeu a $81(48,9 \%)$ pacientes que atenderam aos critérios de seleção.

Na Tabela 1 observa-se que 50,6\% eram do sexo feminino, $54,3 \%$ pertenciam à faixa etária entre 60 a 70 anos, com média de idade de 70,2 anos $( \pm 7,4)$. Quanto à situação conjugal, a maioria era casada (60,5\%) e $24,6 \%$ viúvos. No tocante a religião a maioria era católica $(76,5 \%)$, seguido de protestantes (18,5\%). O grau de escolaridade que predominou foi o ensino fundamental $(81,5 \%)$ e renda familiar mensal variou de 1 a 5 salários (58,0\%).

O tempo em tratamento hemodialítico entre os idosos variou de 1 a 10 anos, com média de 3,5 anos $( \pm 2,2)$. $O$ acesso via corrente sanguínea do tipo Fístula Arteriovenosa (FAV) foi amplamente utilizado para o procedimento de hemodiálise entre os idosos $(82,7 \%)$.

No que concerne aos dados clínicos, a Figura 1 mostra que entre idosos portadores de DRC em hemodiálise, a comorbidade predominante foi a Hipertensão Arterial Sistêmica (HAS) presente em $82,7 \%$ dos pesquisados. Destes $48,1 \%$ apresentavam HAS isoladamente e $34,6 \%$ associada ao Diabetes Mellitus (DM). Observou-se ainda, que $11 \%$ dos idosos tinham como comorbidade o DM isoladamente e 6,2 tinham Insuficiência Cardíaca Congestiva (ICC), caracterizada por comprometimento cardiovascular.

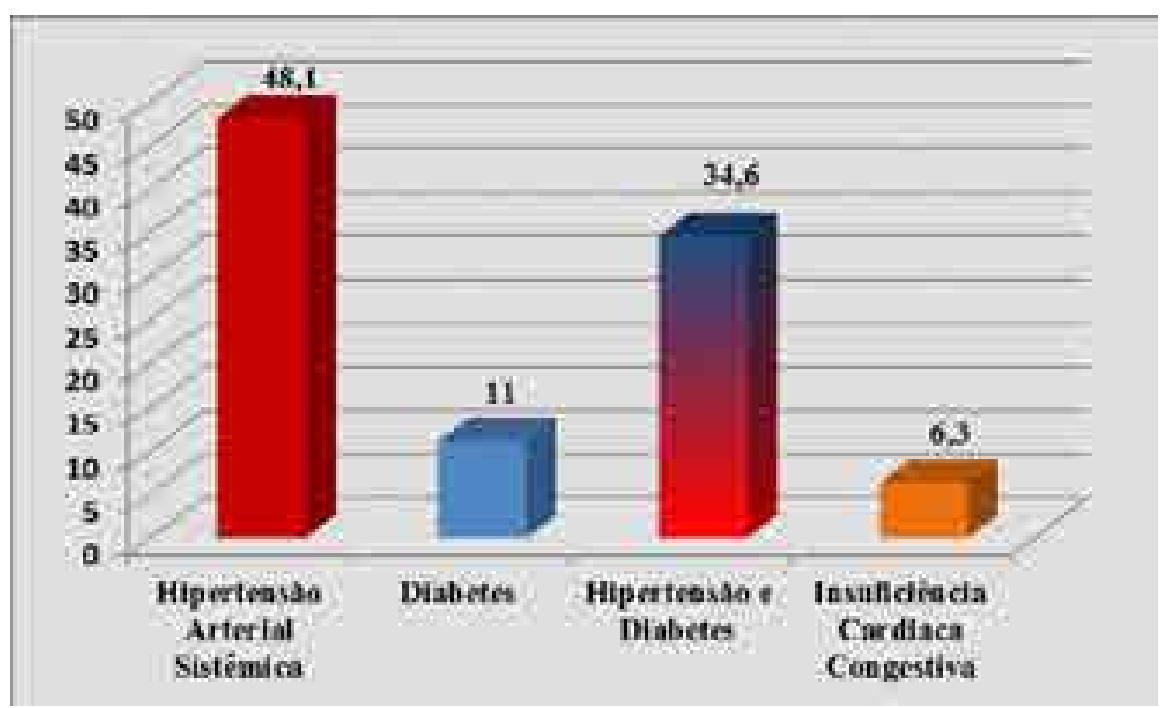

Figura 1 - Distribuição dos idosos em tratamento hemodialítico segundo comorbidades. Natal-RN, 2014. 
Tabela 1 - Distribuição dos dados sociodemográficos dos idosos em tratamento hemodialítico. Natal-RN, 2014

\begin{tabular}{|c|c|c|}
\hline Categorias & $(n=81)$ & $\%$ \\
\hline \multicolumn{3}{|l|}{ Sexo } \\
\hline Masculino & 40 & 49,4 \\
\hline Feminino & 41 & 50,6 \\
\hline \multicolumn{3}{|l|}{ Faixa etária } \\
\hline $60 \mathrm{~A} 70$ & 44 & 54,3 \\
\hline 71 A 80 & 30 & 37,0 \\
\hline 81 A 90 & 07 & 8,7 \\
\hline \multicolumn{3}{|l|}{ Situação conjugal } \\
\hline Solteiro & 02 & 2,5 \\
\hline Casado & 49 & 60,5 \\
\hline Separado & 05 & 6,2 \\
\hline $\begin{array}{l}\text { Desquitado/ } \\
\text { divorciado }\end{array}$ & 05 & 6,2 \\
\hline Viúvo & 20 & 24,6 \\
\hline \multicolumn{3}{|l|}{ Religião } \\
\hline Católico & 62 & 76,5 \\
\hline Protestante & 15 & 18,5 \\
\hline Espirita & 02 & 2,5 \\
\hline Outra & 02 & 2,5 \\
\hline \multicolumn{3}{|c|}{ Grau de escolaridade } \\
\hline $\begin{array}{l}\text { Ensino } \\
\text { fundamental }\end{array}$ & 66 & 81,5 \\
\hline Ensino médio & 02 & 2,5 \\
\hline $\begin{array}{l}\text { Ensino } \\
\text { superior }\end{array}$ & 13 & 16,0 \\
\hline \multicolumn{3}{|c|}{ Renda familiar mensal } \\
\hline$<1$ salário & 04 & 4,9 \\
\hline 1 a 5 salários & 47 & 58,0 \\
\hline 6 a 10 salários & 19 & 23,5 \\
\hline$>10$ salários & 11 & 13,6 \\
\hline \multicolumn{3}{|c|}{ Tempo em hemodiálise } \\
\hline$<1$ ano & 20 & 24,7 \\
\hline 1 a 3 anos & 25 & 30,9 \\
\hline 4 a 6 anos & 17 & 21,0 \\
\hline 7 a 10 anos & 19 & 23,4 \\
\hline \multicolumn{3}{|l|}{ Tipo de Acesso } \\
\hline $\begin{array}{l}\text { Fístula } \\
\text { arteriovenosa }\end{array}$ & 67 & 82,7 \\
\hline Enxerto & 05 & 6,2 \\
\hline $\begin{array}{l}\text { Cateter curta } \\
\text { permanência }\end{array}$ & 08 & 9,9 \\
\hline $\begin{array}{l}\text { Cateter longa } \\
\text { permanência }\end{array}$ & 01 & 1,2 \\
\hline
\end{tabular}

Houve semelhança entre o número de idosos segundo o sexo masculino e feminino, o que diverge dos achados de outros estudos ${ }^{(8-11)}$ realizados com pacientes renais nos quais o sexo masculino foi predominante.

Em estudo realizado ${ }^{(8)}$ para traçar o perfil biopsicossocial de pacientes renais crônicos em tratamento hemodialítico na região sul do Brasil, o sexo masculino predominou e correspondeu a $60,0 \%$ da amostra estudada. O mesmo ocorreu em outro estudo ${ }^{(9)}$ em unidade de hemodiálise no estado de São Paulo, no qual se identificou $61 \%$ do sexo masculino. Autores ${ }^{(10)}$ estudaram as características de pacientes renais crônicos em hemodiálise na região sul do Brasil e identificaram que a maioria dos pacientes também era do sexo masculino (59,3\%).

Outro aspecto relevante é que o número de pessoas idosas no Brasil vem crescendo a cada ano e, consequentemente, há aumento de volume de pacientes que evoluem para DRC e que necessitam de terapias renais substitutivas para sobreviver ${ }^{(12-13)}$. Esses dados foram confirmados pelo censo da Sociedade Brasileira de Nefrologia, realizado em 2011, entre pacientes em hemodiálise com idade igual ou superior a 65 anos $^{(5)}$.

Em relação à situação conjugal dos idosos pesquisados, autores ${ }^{(10-11)}$ obtiveram resultados semelhantes ao estudarem pacientes em hemodiálise, no qual predominaram os casados.

Tratando-se da religião, estudos ${ }^{(11,14)}$ observaram que a espiritualidade pode comprometer a capacidade dos pacientes em lidar com a doença, e ainda, agravar sintomas físicos e emocionais e interferir no desfecho da DRC. Pesquisa na região centro-oeste do Brasil, com 120 pacientes renais crônicos em hemodiálise, obteve resultados semelhantes com esta pesquisa, com percentual de $79,2 \%$ de adeptos a religião católica. Os autores relatam que as questões espirituais ainda são pouco abordadas nos estudos realizados por enfermeiros ${ }^{(14)}$.

Quanto à escolaridade verificou-se semelhança no grau de escolaridade encontrado - ensino fundamental completo e incompleto - entre os idosos desta pesquisa, com estudos feitos na região nordeste e sul, apesar de haver discrepâncias socioeconômicas entre as diferentes regiões do $\operatorname{Brasil}^{(10,15)}$. 
Quanto à renda familiar mensal dos pesquisados foi observada a variação entre menos 1 e 5 salários mínimos, destes 4,9\% apresentavam renda inferior a 1 salário. Vale ressaltar que o salário mínimo nacional vigente no período da coleta de dados era de $\mathrm{R} \$$ 724,00 (setecentos e vinte e quatro reais). O nível socioeconômico baixo é considerado fator de risco para o desenvolvimento de doenças crônicas. No entanto, o aparecimento da DRC está inversamente relacionado ao poder econômico das pessoas e apresenta maior relação com características étnicas ${ }^{(11,16)}$.

O tempo de permanência na modalidade de hemodiálise variou entre um e 10 anos, com média de 3,5 anos $( \pm 2,2)$. Em estudo realizado ${ }^{(10)}$, o tempo de permanência em hemodiálise predominante foi de 2 a 4 anos (30,0\%). No entanto, a soma do número de pacientes de 0 a 4 anos totalizou 53,3\%, aproximando-se dos achados do presente estudo que atingiu $55,6 \%$.

A maioria dos idosos utilizava a FAV, como via de acesso a corrente sanguínea durante as sessões de hemodiálise. A FAV obtida pela junção cirúrgica entre uma artéria e uma veia é considerado o acesso vascular definitivo para hemodiálise ${ }^{(17)}$.

Em pesquisa ${ }^{(18)}$ realizada sobre acesso vascular para hemodiálise em um hospital de Cuba, 66,6\% dos pesquisados utilizaram a FAV como acesso vascular e 33,4\% o cateter de curta permanência. Quanto ao sítio de inserção a veia jugular (20,0\%) foi a mais utilizada, seguido da veia subclávia (13,3\%).

O local ou sítio de inserção do cateter de curta permanência deve ser escolhido cuidadosamente, levando-se em consideração as particularidades anatômicas e clínicas do paciente. O procedimento de implante de cateter é rápido e exige os mesmos cuidados inerentes a qualquer procedimento cirúrgico. Após o implante é preciso confirmar o posicionamento do cateter por radiografia, antes do início da hemodiálise ${ }^{(19)}$.

Atualmente o local de eleição para implante de cateter de curta permanência para hemodiálise é a veia jugular interna, já que a veia subclávia teve seu uso diminuído devido a graves complicações associadas, como o desenvolvimento de trombose venosa e estenose tardia. O tipo de acesso vascular e a existência de infecção influenciam a mortalidade de pacientes em diálise ${ }^{(18)}$.
Resultados semelhantes sobre dados clínicos dessa pesquisa foram encontrados em estudo ${ }^{(10)}$ desenvolvido com 32 pacientes em hemodiálise, no qual obteve-se como comorbidades associadas à DRC a HAS em $21 \%$ e a DM em $11 \%$ dos pesquisados.

Também, a HAS (35\%) e o DM (30\%) foram os diagnósticos de base predominantes entre 100.397 pacientes em diálise, segundo resultados do censo da Sociedade Brasileira de Nefrologia de 2013. Cabe ressaltar que o censo não abrangeu a totalidade de pacientes em diálise no país, pois as informações foram recolhidas de 54,9\% dos centros de nefrologia em funcionamento no Brasil $^{(5)}$.

Estudo realizado com 86.588 indivíduos, com risco elevado para DRC nos Estados Unidos, identificou que $63,3 \%$ eram hipertensos, $27,7 \%$ eram diabéticos e no decorrer de um ano 15\% progrediram para $\mathrm{DRC}^{(20)}$.

A hipertensão arterial pode ocorrer isoladamente durante a sessão de hemodiálise, sendo responsável por 5\% das intercorrências, mas quando somada às arritmias cardíacas, bradicardia e taquicardia corresponde a $8 \%$ das complicações durante os procedimentos hemodialíticos. A fisiopatologia da crise hipertensiva é ainda obscura sendo a indicação de suspender os medicamentos anti-hipertensivos no período pré-dialítico fator contribuinte para a

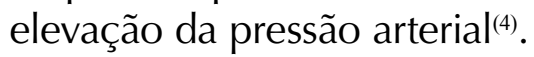

\section{CONCLUSÃO}

A análise dos 81 idosos em tratamento hemodialítico revelou as seguintes características sociodemográficos: a média de idade dos pacientes foi de 70,2 anos $( \pm 7,4)$, a maioria casados; com ensino fundamental; católicos e com renda familiar mensal de menos 1 a 5 salários mínimos. Quanto aos aspectos clínicos, o tempo médio em diálise foi de 3,5 anos $( \pm 2,2)$ e a fístula arteriovenosa o acesso predominante entre os idosos. A hipertensão e o diabetes foram as comorbidades predominantes, seguido de pacientes que tinham as duas enfermidades, simultaneamente.

O envelhecimento populacional, o desenvolvimento de DRC e a dependência de tratamento hemodialítico representam um grande 
desafio aos gestores de saúde, profissionais e familiares. Por isso, o trabalho dos profissionais dos serviços de saúde, principalmente da enfermagem, deve ser direcionado à educação em saúde, com base na prevenção das doenças crônicas não transmissíveis como a DRC.

Conhecer as características sociodemográficas, bem como as características clínicas de pacientes idosos renais crônicos, torna-se importante ferramenta para a equipe de saúde planejar qual a intervenção mais adequada para cada idoso. Além do mais, é preciso que os profissionais de saúde busquem, continuamente, conhecimentos sobre as principais causas de DRC no Brasil.

\section{REFERÊNCIAS}

1. Batista AS, Jaccoiud LB, Aquino L, El Moor PD. Envelhecimento e dependência: desafios para a organização da proteção social. Coleção Previdência Social, Brasília: MPS, SPPS; 2008.

2. Brasil. Lei n. 10.741 , de $1^{\circ}$ de outubro de 2003. Dispõe sobre o Estatuto do Idoso e dá outras providências. Presidência da República, Casa Cívil, Brasília, 1 de out 2003.

3. Moreira V, Nogueira FNN. Do indesejável ao inevitável: a experiência vivida do estigma de envelhecer na contemporaneidade. Psic.-USP [Internet] 2008;19(1). [acesso em 05 abr 2014]. Disponível: http://dx.doi. org/10.1590/S0103-65642008000100009

4. Menezes T, Guimarães E, Machado S, Santos D. Clinical and social profile of users with arterial hypertension. J Nurs Ufpe online. [Internet] 2012; [cited 2014 abr 05]; 6(9). Available from: http://www. revista.ufpe.br/revistaenfermagem/index.php/revista/ article/view/2792

5. Sociedade Brasileira de nefrologia. Censo 2013. [acesso em 10 nov 2014]; Disponível: http://www.sbn. org.br/pdf/censo_2013-14-05.pdf

6. Dutra MC, Uliano EJM, Machado DFGP, Martins T, Schuelter-Trevisol F, Trevisol DJ. Avaliação da função renal em idosos: um estudo de base populacional. J. bras. nefrol. [Internet] 2014;36(3) [acesso em 11 nov 2014]. Disponível: http://dx.doi.org/10.5935/01012800.20140043

7. Ministério da Saúde (BR). Conselho Nacional de Saúde. Diretrizes e normas regulamentadoras de pesquisas envolvendo seres humanos. Resolução n. 466, de 12 de dezembro de 2012. Brasília; 2012.

8. Lanza AHB, Chaves APA, Garcia RCP, Brandão
JAG. Perfil biopsicossocial de pacientes renais crônicos em tratamento hemodialítico. Arq. Bras. Cienc. Saude [Internet] 2008;33(3) [acesso em 05 abr 2014]. Disponível: http://files.bvs.br/ upload/S/1983-2451/2008/v33n3/a141-145.pdf

9. Ribeiro RCHM, Ferrari RR, Bertolin DC, Canova JCM, Lima LCEQ, Ribeiro DF. O perfil sócio-demográfico e as principais complicações intradialíticas entre pacientes com insuficiência renal crônica em hemodiálise. Arq. Cienc. Saude [Internet] 2009;16(4) [acesso em 05 abr 2014]. Disponível: http://www.cienciasdasaude. famerp.br/racs_ol/vol-16-4/IDK6_out-dez_2010.pdf

10. Kirchner RM, Löbler LL, Machado RF, Stumm EMF. Characterization of patients with chronic renal insufficiency in hemodialysis. J Nurs Ufpe online. 2011;5(2):199-204.

11. Takemoto AY, Okubo P, Bedendo J, Carreira L. Avaliação da qualidade de vida em idosos submetidos ao tratamento hemodialítico. Rev. gauch. enferm. [Internet] 2011;32(2) [acesso em 05 abr 2014] Disponível: http://dx.doi.org/10.1590/S198314472011000200007

12. Bastos RAA, Fernandes MGM, Soares MJO, Costa MML, Sousa FS, Almeida FCA. Physiological adaptation of elderly in hemodialysis treatment: an analysis in the light of the Roy Model. J Nurs Ufpe online [Internet] 2014;8(4) [cited 2014 abr 05]. Available from: http:// www.revista.ufpe.br/revistaenfermagem/index.php/ revista/article/view/4743

13. Tavares DMS, Dias FA, Santos NMF, Hass VJ, Miranzi SCS. Factors associated with the quality of life of elderly men. Rev. Esc. Enferm. USP [Internet] 2013;47(3) [cited em 2014 abr 05]. Available from: http://dx.doi. org/10.1590/S0080-623420130000300022

14. Chaves ECL, Carvalho EM, Terra SF, Souza L. Validação clínica de espiritualidade prejudicada em pacientes com doença renal crônica. Rev. Latino-Am. Enfermagem [Internet] 2010;18(3) [acesso em 05 abr 2014]. Disponível: http://dx.doi.org/10.1590/S010411692010000300003

15. Lenardt $\mathrm{MH}$, Hammerschmidt KSA, Modesto Ap, Borghi ACS. O sistema de conhecimento e de cuidado dos idosos em hemodiálise concernente a terapia medicamentosa. Cogitare enferm. [Internet] 2008;13(2) [acesso em 02 set 2014]. Disponível: http://dx.doi. org/10.5380/ce.v13i2.12432

16. Zambonato TK, Thomé FS, Gonçalves LFS. Perfil Socioeconômico dos pacientes com doença renal crônica em diálise na região noroeste do Rio Grande do Sul. J. bras. nefrol. [Internet] 2008;30(3) [acesso em 05 abr 2014]. Disponível: http://www.jbn.org.br/ detalhe_artigo.asp?id=57

17. Freitas C, Silva F, Matos N, Machado R, Queirós J, 
Almeida R, et al. Consulta de acessos vasculares para hemodiálise - experiência de um centro angiologia e cirurgia vascular. Angiol cir vasc [Internet] 2011;7(1) [acesso em 05 abr 2014]. Disponível: http://www. scielo.gpeari.mctes.pt/pdf/ang/v7n1/v7n1a05.pdf

18. Rojas EJJ, Alfonso SF, Castelo VX, Semanat VD. Calidad del modelo de gestión actual del acceso vascular en los pacientes en hemodiálisis. Rev. cuba. med. [Internet]. 2012;51(2) [citado 2014 nov 11]. Disponible en: http:// scielo.sld.cu/scielo.php?script=sci_arttext\&pid=S0034$75232012000200002 \& \operatorname{lng}=\mathrm{es}$

19. Torres GV, Santos SCLC, Mendonça AEO, Barreto AFG, Costa IKF, Melo GSM. Incidência de infecção em pacientes com cateter temporário para hemodiálise. Rev enferm UFPE on line. 2010; 4(1):170-7.

20. Jurkovitz CT, Li S, Norris KC, Saab G, Bombak AS, Whaley-Connell AT, et al. Association between lack of health insurance and risk of death and ESRD: results from the Kidney Early Evaluation Program (KEEP). Am J Kidney Dis. 2013;61(4 Suppl 2):24-32. 